\title{
(+)-BINOL and Pure Shift Experiment: A Bidirectional Approach for NMR Chiral Discrimination of Overcrowded Spectra of Primary Amines
}

\author{
Juliana C. Merino, ${ }^{a}$ Artur F. Keppler ${ }^{a}$ and Márcio S. Silva ${ }^{*, a}$ \\ ${ }^{a}$ Centro de Ciências Naturais e Humanas (CCNH), Universidade Federal do ABC (UFABC), \\ 09210-380 Santo André-SP, Brazil
}

\begin{abstract}
In this work, enantiopure 1,1'-bi-2-naphthol (BINOL) derivatives and carboxylic acids were evaluated as chiral solvating agents (CSAs) in the nuclear magnetic resonance (NMR) chiral discrimination of primary amines. Among the CSAs screened, the readily available (+)-BINOL revealed a simple and rapid chiral auxiliary for ${ }^{1} \mathrm{H}$ NMR chiral discrimination. The procedure required few minutes, assigning of the absolute configuration. The protocol was improved using the ${ }^{1} \mathrm{H}$ pure shift pulse sequence for overcrowded alkyl primary amines due to the absence of $J_{\mathrm{HH}}$ couplings. The ${ }^{1} \mathrm{H}$ pure shift NMR experiment and the readily available (+)-BINOL chiral auxiliary can be used for routine chiral discrimination analysis of complex NMR spectra.
\end{abstract}

Keywords: NMR chiral discrimination, primary amines, pure shift experiment, chiral solvating agent

\section{Introduction}

Chiral compounds play a major role in the pharmaceutical industry and in asymmetric synthesis. ${ }^{1-3}$ Over the last few decades, chirality has changed the methodologies associated with the synthesis of drug molecules, together with the distinction of biological properties. Among organic compounds employed in the pharmaceutical industry, chiral amines have high demand due to their broad spectrum of biological activities and to their application as chiral building blocks. ${ }^{4-9}$

Facile and fast methods for chiral recognition are in constant development. Chiral chromatography is still the most applied analytical method for determination of the enantiomeric excess (ee) of chiral compounds. ${ }^{10-12}$ The main advantages of this technique are high resolution and the use of a trace amount of sample. However, limitations include a lack of green chemistry features, as well as long times for analysis, toxic solvents, expensive columns and inefficiency in resolving volatile or thermally unstable compounds.

Nowadays, nuclear magnetic resonance (NMR) spectroscopy is an alternative technique for the determination of enantiomeric purity and for the determination of absolute configuration. ${ }^{13,14}$ The method is based on converting

*e-mail: s.marcio@ufabc.edu.br enantiomers to diastereoisomers. The classical approach is to use a chiral auxiliary, which can be a chiral derivatizing agent (CDA), chiral solvating agent (CSA) or chiral lanthanide shift reagent (CLSR). The success of NMR chiral recognition can be observed by the increasing number of new methods based on it. ${ }^{15-19}$

The use of established commercial compounds, such as Mosher's acid (MTPA), as chiral auxiliaries is not a practical alternative, because MTPA requires a time consuming procedure for derivatization ${ }^{20}$ and has low reactivity ${ }^{21}$ high hygroscopic and poor resolution ability in NMR caused by the presence of multiple conformers. ${ }^{22}$ Most of the times, the multistep synthesis of a chiral auxiliary involves the presence of impurities, purification procedures and time-consuming, discouraging the use of NMR as a strategic route for chiral recognition of enantiomers and determination of their composition.

Recently, new protocols, ${ }^{23-26}$ reagents $^{27,28}$ and NMR pulse sequences ${ }^{29,30}$ were developed for simple and fast NMR chiral recognition. Among chiral auxiliaries, 1,1'-bi-2-naphtol (BINOL) represents a useful auxiliary for chiral recognition..$^{31}$ The BINOL reagent and derivatives have been employed directly as a CSA for NMR chiral discrimination of isoflavones, ${ }^{32}$ flavones ${ }^{33}$ sulfinimines, ${ }^{34}$ omeprazole $^{\circledR},{ }^{35}$ alkaloid crispine $\mathrm{A}^{36}$ and promethazine. ${ }^{37}$ In addition, the combination of simple chiral auxiliaries with practical protocols and/or high resolution NMR 
experiments (bidirectional approach) ${ }^{13}$ provides an elegant strategy to overcome overlapping signals and degradation problems.

Herein, we report a simple and efficient NMR chiral discrimination of primary amines, amino alcohols and amino selenides using (+)-BINOL as a CSA and ${ }^{1} \mathrm{H}$ pure shift NMR experiment to improve the resolution of overcrowded spectra due to the absence of $J_{\mathrm{HH}}$ couplings.

\section{Experimental}

NMR experiments were recorded in a Varian Unityplus $500 \mathrm{MHz}$ in a $5 \mathrm{~mm}$ direct broadband observe probe. The ${ }^{1} \mathrm{H}$ NMR spectra were acquired using 8 scans, $1 \mathrm{~s}$ of relaxation time and $4.67 \mathrm{~s}$ of acquisition time at $27^{\circ} \mathrm{C}$. For processing the ${ }^{1} \mathrm{H}$ NMR spectra, $0.3 \mathrm{~Hz}$ of line broadening and $64 \mathrm{k}$ of points were used. The ${ }^{1} \mathrm{H}$ pure shift NMR experiments were acquired using the pure shift 1D pulse sequence from the Agilent Technologies Inc. 2014 (VNMRJ 4.2) (38 $^{38}$ with 64 scans, $1.68 \mathrm{~s}$ of acquisition time, $2 \mathrm{~s}$ of relaxation delay, $10.93 \mu \mathrm{s}$ of pulse width $\left(90^{\circ}\right), 60 \mathrm{~ms}$ of slice selection bandwidth (bandwidth for selective pulse) and $10 \mathrm{~Hz}$ for the coupling constant $(J)$ delay (delay for effective homonuclear decoupling).

The 2D heteronuclear single-quantum correlation, HSQC-edit spectra, were recorded using 16 scans, 16k of acquisition points, 400 increment resolution, $1.5 \mathrm{~s}$ of relaxation delay and $10.93 \mu$ s of pulse width $\left(90^{\circ}\right)$. The $J$-resolved spectrum was acquired using 64 scans, $64 \mathrm{~Hz}$ of spectral window, $4 \mathrm{k}$ of acquisition points, $1.5 \mathrm{~s}$ of relaxation delay and $10.93 \mu \mathrm{s}$ of pulse width $\left(90^{\circ}\right)$. The total correlated spectroscopy (TOCSY) and Z-TOCSY (pulse sequence from Agilent Technologies Inc. 2014-VNMRJ 4.2) NMR experiments were recorded using 16 scans, $4 \mathrm{k}$ of acquisition points, 400 increment resolution, $1.5 \mathrm{~s}$ of relaxation delay, $10.93 \mu \mathrm{s}$ of pulse width $\left(90^{\circ}\right)$ and $150 \mathrm{~ms}$ of spin-lock time.

All reagents and solvents were obtained from commercial suppliers (Sigma-Aldrich) and were used without further purification. The samples for NMR spectroscopy were prepared by weighing and dissolving $0.05 \mathrm{mmol}$ of the racemic primary amine with $0.1 \mathrm{mmol}$ of (+)-BINOL in $500 \mu \mathrm{L}$ of deuterated chloroform $(0.03 \%$ $\mathrm{v} / \mathrm{v}$ of tetramethylsilane, TMS) in a $5 \mathrm{~mm}$ NMR tube. The solutions were shaken for 2 min for equilibration.

\section{Results and Discussion}

Initially, six types of readily available CSAs $1 \mathbf{a}-\mathbf{1 g}$ were evaluated using racemic methylbenzylamine (MBA) 2a as a model substrate, employing 1.0 equiv. of each compound in $\mathrm{CDCl}_{3}$ at $27^{\circ} \mathrm{C}$ (Figure 1). Mixtures of racemic MBA 2a with the CSAs 1a and 1c did not display chemical shift differences. The CSA 1c induced no anisotropy, possibly because of the steric hindrance of 3,5-methylphenyl groups. When the CSAs 1d and 1e were employed, a precipitate was formed. The white solid of CSA 1e was obtained faster than $1 \mathbf{d}$ due to the stronger ionic interaction between the reagents, since the $O$-acetyl mandelic acid did not show this precipitate. ${ }^{39}$ The Mosher acid $\mathbf{1 f}$ resulted in a magnetic anisotropic effect on the substrate $\mathbf{2 a}(\Delta \delta 0.05 \mathrm{ppm})$. However, the (+)-BINOL $\mathbf{1 b}$ presented a more satisfactory result $(\Delta \delta 0.10 \mathrm{ppm})$.

After the CSA screening, the influence of the amount of (+)-BINOL 1b on chemical shift anisotropy was investigated. The addition of 0.5 equiv. $(\Delta \delta 0.06 \mathrm{ppm})$ decreased the signal separation, and 2.0 equiv. ( $\Delta \delta 0.16 \mathrm{ppm})$ increased this value. The addition of 3.0 or more equivalents did not result in improvements in the split signals. The gradual increase of the amount of (+)-BINOL $\mathbf{1 b}$ resulted in shielding of ${ }^{1} \mathrm{H}$ NMR signals. The shielding effect could be observed in the protons of methine (CH; Figure S4, Supplementary Information (SI) section) and methyl $\left(\mathrm{CH}_{3}\right.$; Figure $\mathrm{S} 4$, SI section) groups. The enantiodiscrimination ability of the CSA (+)-BINOL $\mathbf{1 b}$ was also verified in other solvents like $\mathrm{CD}_{3} \mathrm{CN}$, MeOD- $d_{4}$ and DMSO- $d_{6}$. The results suggest that chloroform is a better solvent, which may be due to protic solvents interfering in hydrogen-bond formation and chloroform has a lower polarity which decreases the solventsubstrate interaction. Once evaluated the amount of reagents ratio and the solvent, the effect of ratio concentration was also investigated, because it is known that higher concentrations usually enhance the chemical shift anisotropy for chiral solvating agent performance. In this way, concentrations above $10 \mathrm{mmol} \mathrm{L}^{-1}$ have shown better $\Delta \delta$ results. In order to evaluate the method, the amine 2a was derivatizated to acetyl amide and a chiral high performance liquid chromatography (HPLC) analysis was carried out to confirm the enantiomers ratio (Figure S5, SI section). The derivatization step was necessary due the low resolution employing directly the primary amine $\mathbf{2 a}$.

When the scope of the protocol is expanded for other racemic primary amines to obtain more information about the NMR resolution behavior using the CSA $\mathbf{1 b}$, the limitations of the simple ${ }^{1} \mathrm{H}$ NMR experiment are presented (Figure 2). The results evidently validated its utility for discrimination on arylalkylamines, as signal separation obtained by NMR chiral discrimination using only the (+)-BINOL $\mathbf{1 b}$ was comparable with other protocols which require more complex procedures. ${ }^{40-45}$ When evaluating these results, the greater separation of arylalkylamines $\mathbf{2 a}, \mathbf{2} \mathbf{b}$, $\mathbf{2 c}$ and $\mathbf{2 f}$ compounds occurred due to steric hindrance and 


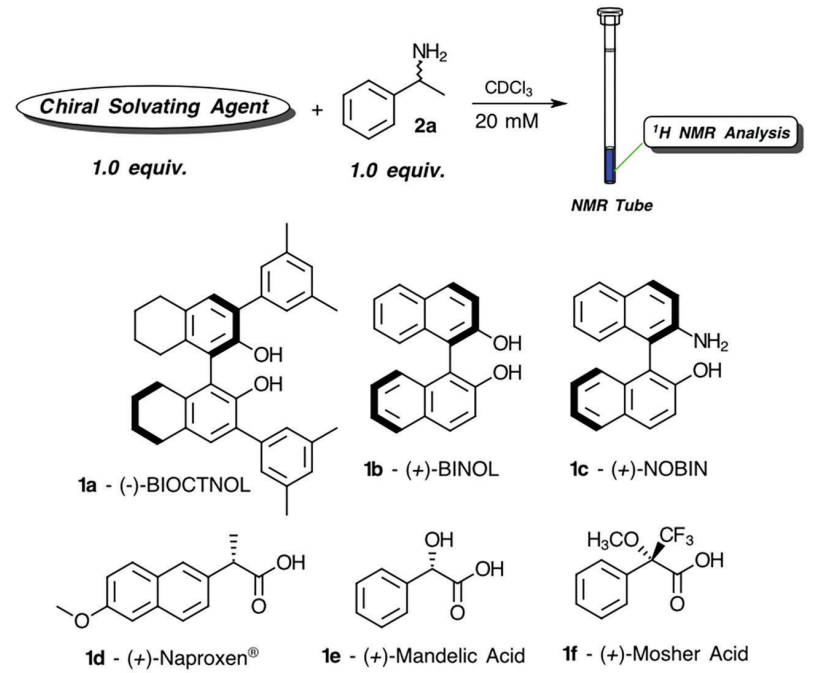

Figure 1. Screening of NMR chiral discrimination ability of chiral solvating agents 1a-g.

chemical shift anisotropy caused by the aromatic ring. The influence of the aromatic ring in the substrates evaluated is supported by the smaller split signals using the alkylamines $\mathbf{2 d}, \mathbf{2 e}, \mathbf{2 g}, \mathbf{2 h}$ and $\mathbf{2 j}$. The deuterated benzene was also verified as a solvent with the alkylamines and the chemical shift anisochrony in the proton spectra was improved only with the compounds $\mathbf{2 e}$ and $\mathbf{2} \mathbf{j}$ (Figures S21 and S22, SI section). The ${ }^{1} \mathrm{H}$ NMR spectra of the diastereomeric interactions examined in the alkyl amines showed that the more distinctive signs were observed in the methyl group $\left(\mathrm{H}_{2} \mathrm{~N}-\mathrm{CH}-\mathrm{CH}_{3}\right)$. Considering these observations, the results indicate that the CSAs are more sensitive to structure variations, like with the chiral primary amine $\mathbf{2 g}$, where the stronger selenium-oxygen ${ }^{46}$ interaction significantly moved the chemical shifts $(\delta)$, resulting in the overlap of some signals (Figure S25, SI section).

After demonstrating the potential of the (+)-BINOL 1b for a simple and faster chiral discrimination, it was extended to the assignment of the absolute configuration
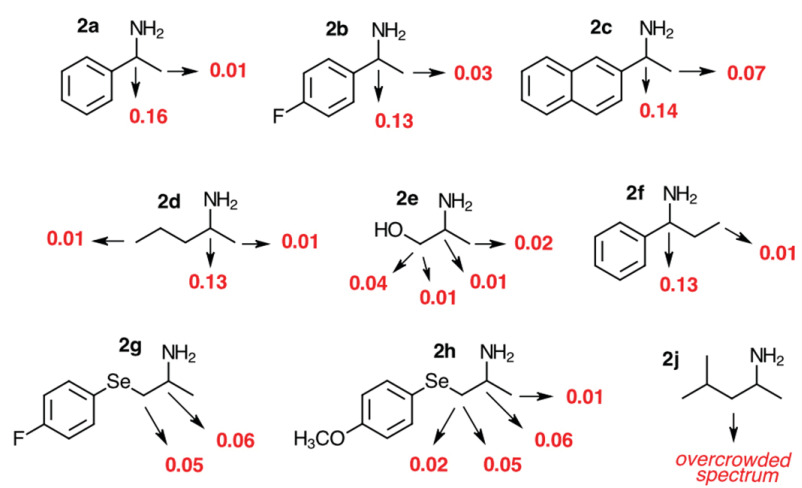

Figure 2. Ability of CSA $\mathbf{1 b}$ to discriminate signals of chiral primary amines 2 in ${ }^{1} \mathrm{H}$ NMR experiments (500 MHz). The red numbers are the chemical shift differences $(\Delta \delta)$ between the enantiomers. of chiral primary amines $\mathbf{2}$. For this purpose, the amines $(S)-(+)$-MBA 2a and $(R)-(-)-$ MNA $2 \mathbf{c}$ were employed. The enantiomerically enriched amines $\mathbf{2 a}$ and $\mathbf{2 c}$ showed the same three-dimensional structural profile, where the methine $\mathrm{CH}$ group of the $(S)$ enantiomer was shielded and the methyl $\mathrm{CH}_{3}$ group was deshielded (Figure 3). For the $(R)$ enantiomer, the inverse chemical shift anisotropy effect was observed (Figure 3). The determination of the absolute configuration of aryl alkyl primary amines could be carried out with substantial differences in chemical shifts due to stronger magnetic anisotropic effect of binaphthyl group, which the smaller groups $\left(\mathrm{CH}\right.$ and $\left.\mathrm{CH}_{3}\right)$ felt the effect of the binaphthyl group of (+)-BINOL $\mathbf{1 b}$.

Initially, six types of easily available CSAs 1a-1g were evaluated for NMR chiral discrimination of primary amines, of which (+)-BINOL $\mathbf{1 b}$ demonstrated the best performance. The efficiency of this stable and ready available chiral compound is also visualized by the possibility to determine the absolute configuration. However, spectra with excessive line broadening and complex multiplicity patterns restricted the chiral analysis using the conventional $1 \mathrm{D}{ }^{1} \mathrm{H}$ NMR spectrum. To resolve these overlapped peaks without the use of multistep syntheses or classical methodologies, which are time- and reagent-consuming, different NMR pulse sequences are possible, such as two dimensional selective $\mathrm{F}_{1}$ decoupling, ${ }^{47,48}{ }^{\mathrm{RES}}$-TOCSY ${ }^{49}{ }^{49} \mathrm{Z}-\mathrm{COSY}^{50}$ and pure shift. ${ }^{30,51}$

The NMR chiral discrimination of the primary amines $\mathbf{2 d}, \mathbf{2} \mathbf{e}, \mathbf{2 g}, \mathbf{2} \mathbf{i}$ and $\mathbf{2 j}$ were used as substrate models to demonstrate the efficiency of specialized high-resolution NMR experiments to overcome signal overlapping. The arylalkylamines $\mathbf{2 a}, \mathbf{2 b}, \mathbf{2 c}$ and $\mathbf{2 f}$ were not used in this evaluation because the success to discriminate the enantiomers by simple ${ }^{1} \mathrm{H}$ NMR.

In order to discriminate the enantiomers, the primary selenide amine $2 \mathrm{~g}$ was employed for 2D NMR analysis. HSQC-edited and $J$-resolved 2D NMR experiments were recorded to assess the diastereoisomer peaks (Figure 4). The measurement of enantiomeric excess using the $J$-resolved 2D NMR experiment could be non-accurate due the proximity of the signals (Figure S18, SI section). On the other hand, the 2D HSQC-edited experiment successfully solved the overlapping of signals, because the methine $\mathrm{CH}$ proton has a different carbon correlation from the methylene $\mathrm{CH}_{2}$ protons (Figure 4). In addition, we applied a simple integral deconvolution (Figure S19, SI section) of the diastereomer signals to provide a precise evaluation of integral values and compare the values with the HSQC-edited NMR experiment.

A TOCSY NMR experiment usually serves as a good technique to differentiate overcrowded signals. Thus, the 


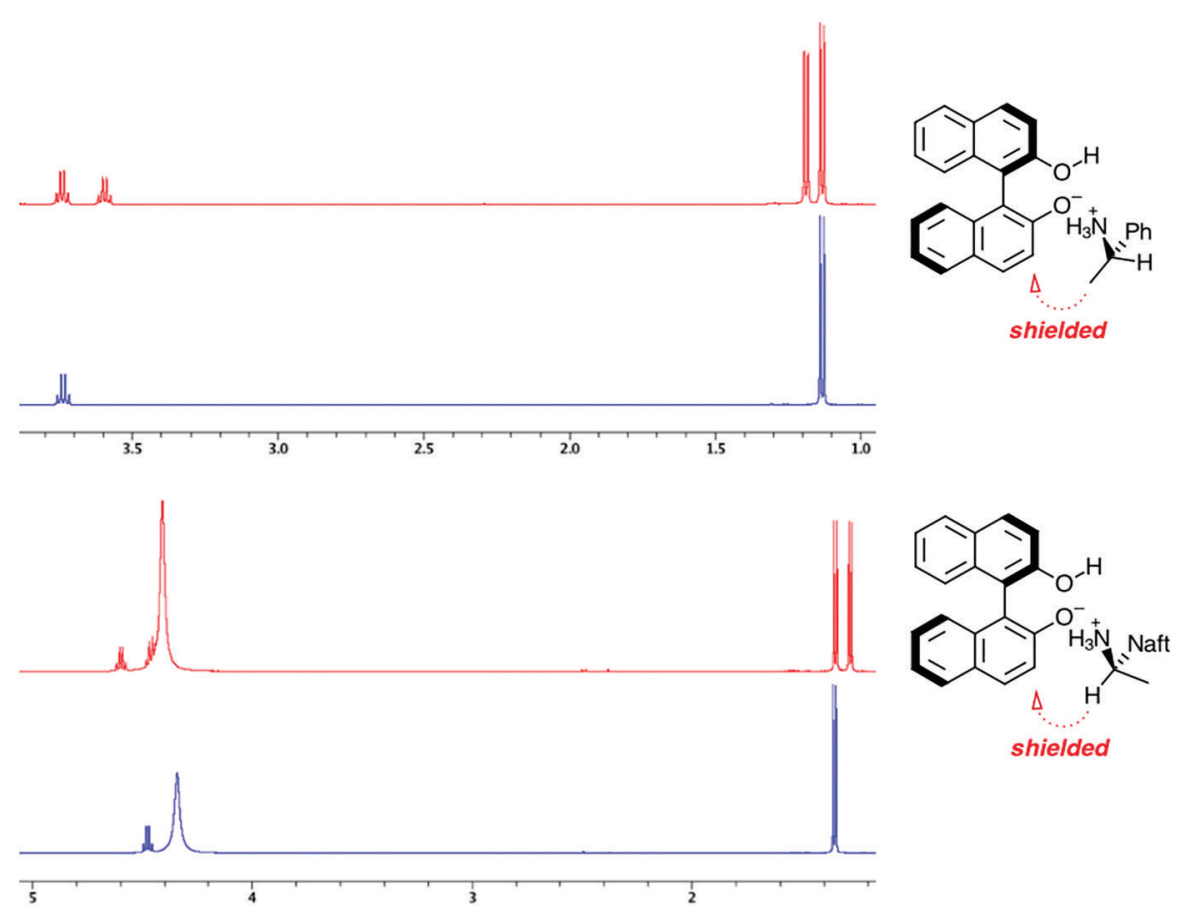

Figure 3. Absolute configuration of primary amines (S)-(+)-MBA 2a (top) and $(R)-(-)-\mathrm{MNA} \mathbf{2 c}$ (bottom) with 2.0 equiv. of (+)-BINOL $1 \mathbf{b}$ in $\mathrm{CDCl}_{3}$ at $27^{\circ} \mathrm{C}$.

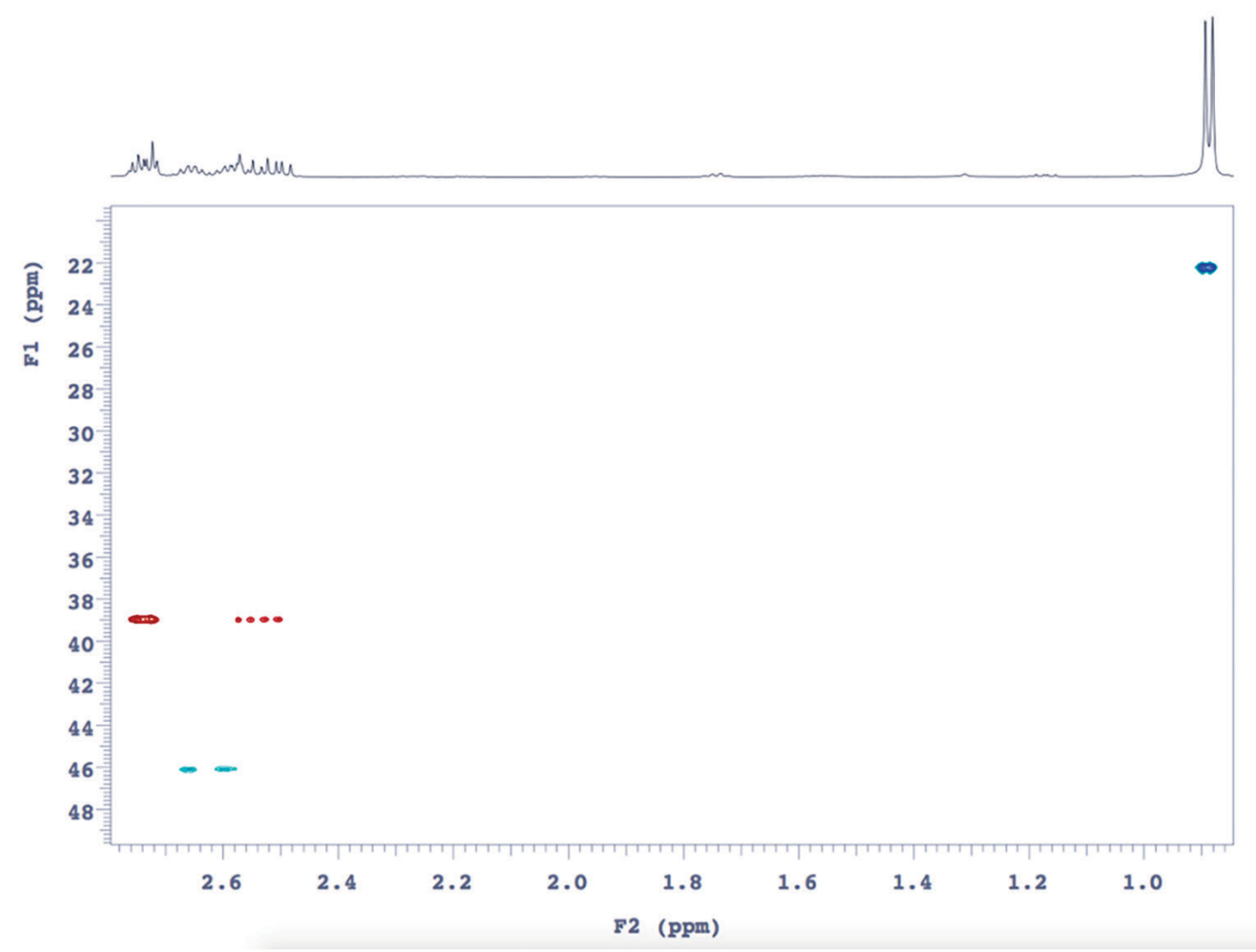

Figure 4. 2D HSQC-edited NMR experiment $\left(500 \mathrm{MHz}, \mathrm{CDCl}_{3}\right)$ of chiral discrimination of selenide amine $\mathbf{2 g}$ using 2.0 equiv. of $(+)$-BINOL $\mathbf{1 b}$ at $27^{\circ} \mathrm{C}$.

next step is to apply the TOCSY related experiments to identify the diastereomers peaks. Using TOCSY experiment was possible to recognize the diastereomers peaks of the primary amine 2d, since the HSQC-edited 2D NMR did not discriminate the signals (Figure S20, SI section). Moreover, the conventional TOCSY pulse sequence and Z-filter variation were recorded to demonstrate the performance of the zero-quantum coherence suppression (Figure 5).

For the overcrowded spectra of primary amines $\mathbf{2 e}$, $2 \mathbf{i}$ and $\mathbf{2 j}$, the chiral discrimination was not achievable 

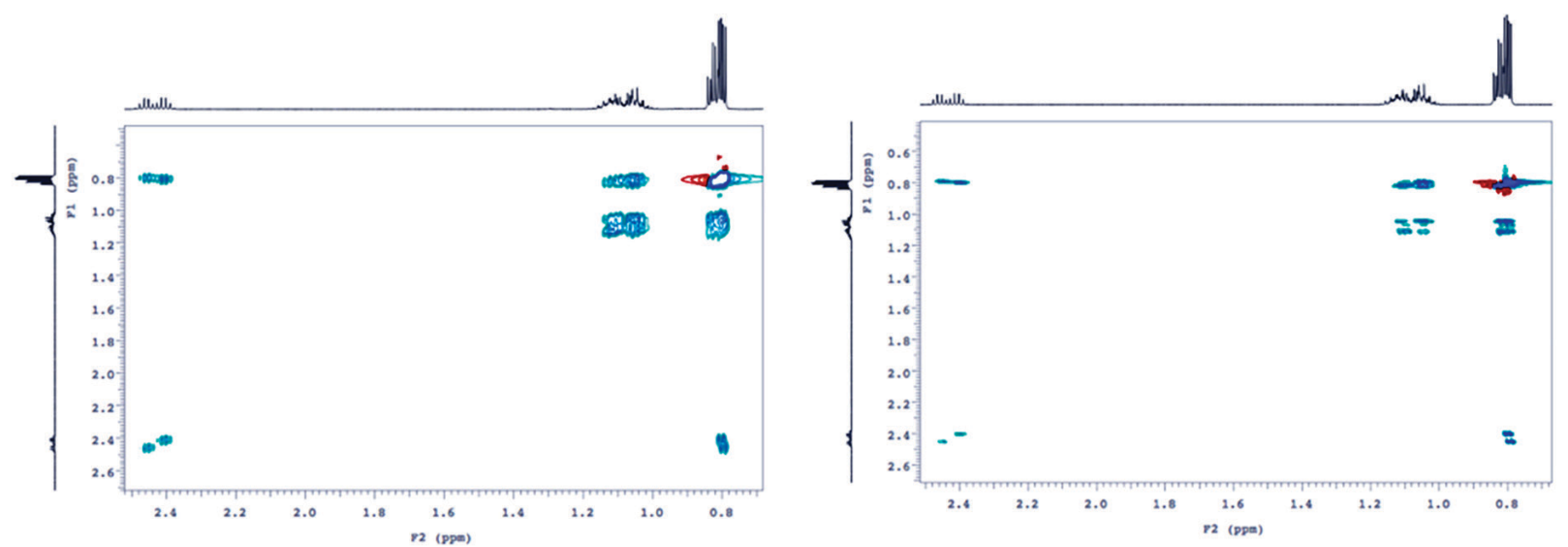

Figure 5. NMR resolution difference between TOCSY (left) and Z-TOCSY (right) NMR experiments (500 MHz, $\mathrm{CDCl}_{3}$ ) of chiral discrimination of the primary amine $2 \mathbf{d}$ using 2.0 equiv. of (+)-BINOL $\mathbf{1 b}$ at $27^{\circ} \mathrm{C}$.

employing these 2D NMR experiments. In these cases, the $1 \mathrm{D}^{1} \mathrm{H}$ pure shift NMR technique was used to discriminate the overlapped peaks. It is possible to observe that the overcrowded signals become clear due to the elimination of scalar coupling, and chiral discrimination could be easily performed (Figure 6). For the primary amine $2 d$, the $1 \mathrm{D}^{1} \mathrm{H}$ pure shift NMR experiment was performed in benzene- $d_{6}$. Sometimes the application of larger spectral window does not allow an efficient homonuclear decoupling, being necessary to use a specific spectral window to remove the scalar couplings. To improve the performance of the pure shift NMR spectra the coupling constant for elimination, spectral window, spectral resolution and the flip angle must be optimized. Moreover, pulse sequence variations could be applied to eliminate the artefacts that usually decrease the quality of the spectra.

The main cost to pay for obtaining a broadband homodecoupled spectrum is the sensitivity. This is a result of the use of selective inversion elements as a part of the $J$-refocusing procedure. The spectral quality of both NMR chiral discrimination analyses could be visualized with proper baseline resolution and no artifacts, which permitted the measurement of the ee of the primary amines $2 \mathbf{e}, 2 \mathbf{i}$ and $\mathbf{2 j}$ (Figure 6). Finally, there are other NMR experiments that can be used to simplify the multiplicity, such as homonuclear decoupling (HOMODEC), which decouples

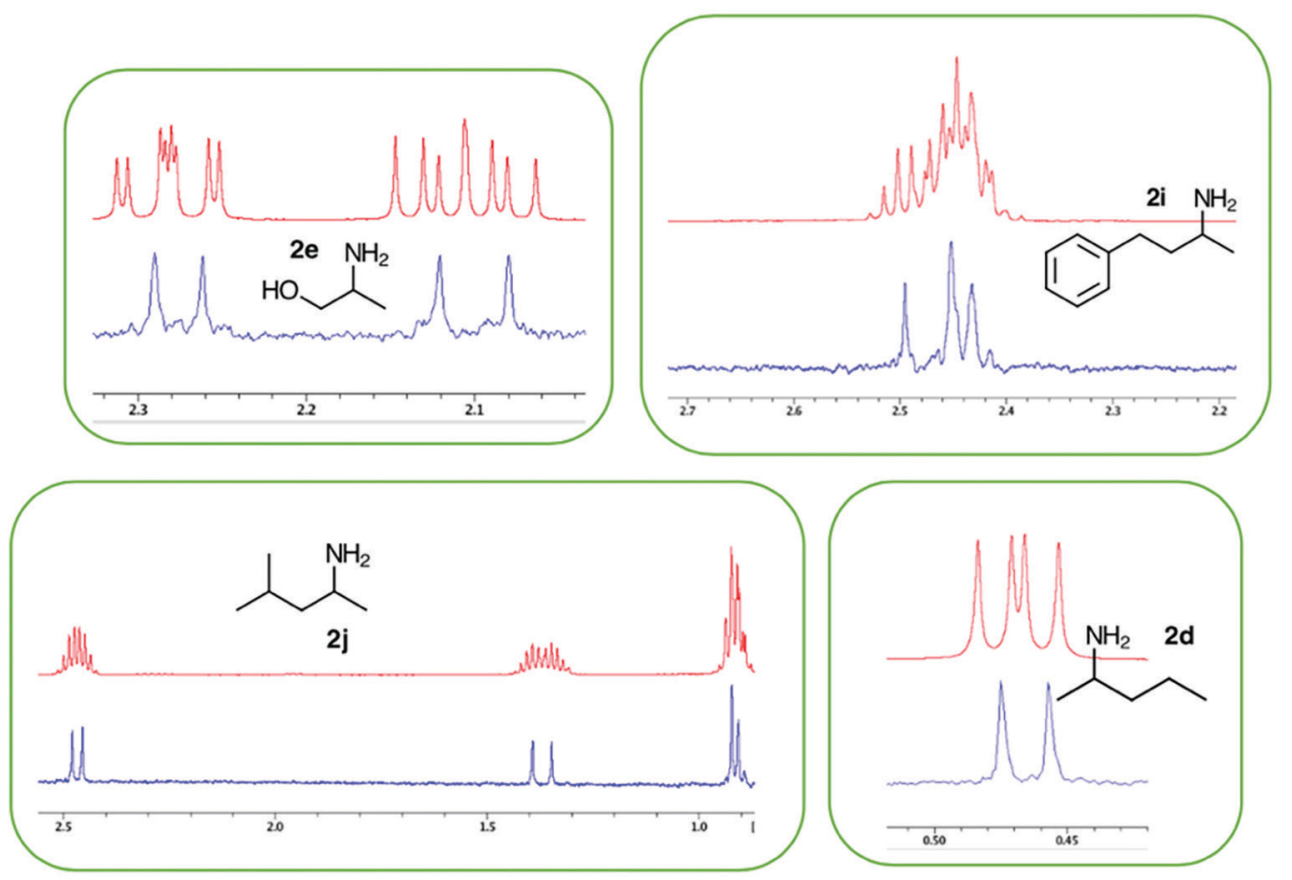

Figure 6. $1 \mathrm{D}^{1} \mathrm{H}$ NMR pure shift experiments $(500 \mathrm{MHz}$ ) for chiral discrimination of the amino alcohol $\mathbf{2 e}$ (left), the primary amine $\mathbf{2 i}$ (right) and primary amine $2 \mathbf{j}$ (down) employing 2.0 equiv. of (+)-BINOL $\mathbf{1 b}$ in $\mathrm{CDCl}_{3}$ at $27^{\circ} \mathrm{C}$. The $1 \mathrm{D}{ }^{1} \mathrm{H}$ pure shift experiment for chiral discrimination of primary amine 2d was performed in benzene- $d_{6}$ at $27{ }^{\circ} \mathrm{C}$. Lower spectrum is the ${ }^{1} \mathrm{H}$ pure shift experiment and upper spectrum is the conventional ${ }^{1} \mathrm{H}$ NMR experiment. 
one coupling partner by selective irradiation. The advantage of HOMODEC compared with pure shift is the use of lowmolarity samples in a shorter time. However, HOMODEC cannot be used for the simplification of all spectral complexity and very similar chemical shifts.

\section{Conclusions}

In conclusion, 2.0 equiv. of (+)-BINOL, a non-hygroscopic, stable to racemization and readily available compound, can be used as a chiral solvating agent for chiral discrimination and the assignment of the absolute configuration of primary amines. However, there are severe limitations using only the ${ }^{1} \mathrm{H}$ NMR experiment. We have demonstrated some alternatives to overcome the overlapping signals. The $1 \mathrm{D}^{1} \mathrm{H}$ pure shift NMR experiment has revealed to be essential for overcrowded peaks. This ultra high resolution pulse sequence is a practical alternative to discriminate enantiomers of drugs candidates and other useful molecules with complex patterns, without multistep synthesis and purification steps.

\section{Supplementary Information}

The experimental details and spectra of ${ }^{1} \mathrm{H}$ NMR, ${ }^{1} \mathrm{H}$ pure shift NMR and 2D NMR (HSQC-edited and $J$-resolved) experiments are available free of charge at http://jbcs.sbq.org.br as a PDF file.

\section{Acknowledgments}

The authors thank the Central Experimental Multiusuário-CEM/UFABC for NMR equipment.

\section{References}

1. Morris, D. G.; Stereochemistry; The Royal Society of Chemistry: Cambridge, 2001.

2. Robinson, J. J. T.; Organic Stereochemistry; Oxford Chemistry Primers Series, Oxford University Press: New York, 2001.

3. Tao, J.; Lin, G.; Liese, A.; Biocatalysis for the Pharmaceutical Industry: Discovery, Development and Manufacturing; John Wiley \& Sons Ltd.: Singapore, 2009.

4. Cary, J. S.; Laffan, D.; Thomson, C.; Williams, M. T.; Org. Biomol. Chem. 2006, 4, 2337.

5. Gotor, V.; Cerrada, S. G.; Mendiola, J.; Frutos, O.; Collado, L.; Fernández, V. G.; Lavandera, I.; Busto, E.; Mata, M. R.; Paul, C. E.; Org. Process Res. Dev. 2014, 18, 788.

6. Krasnov, V. P.; Gruzdev, D. A.; Levit, G. L.; Eur. J. Org. Chem. 2012, 1471.

7. Nugent, T. C.; El-Shazly, M.; Adv. Synth. Catal. 2010, 352, 753.
8. Skucas, E.; Ngai, M.-Y.; Komanduri, V.; Krische, M. J.; Acc. Chem. Res. 2007, 40, 1394.

9. Muller, T. E.; Hultzsch, K. C.; Yus, M.; Foubelo, F.; Tada, M.; Chem. Rev. 2008, 108, 3795.

10. Shen, J.; Okamoto, Y.; Chem. Rev. 2016, 116, 1094.

11. Okamoto, Y.; Ikai, T.; Chem. Soc. Rev. 2008, 37, 2593.

12. Bi, C.; Zheng, X.; Azaria, S.; Beeram, S.; Li, Z.; Hage, D. S.; Separations 2016, 3, 27.

13. Mishra, S. K.; Chaudhari, S. R.; Lakshmipriya, A.; Pal, I.; Lokesh, N.; Suryaprakash.; Annual Reports on NMR Spectroscopy, vol. 91; Webb, G. A., ed.; Academic Press: London, 2017, ch. 4.

14. Yang, L.; Wenzel, T.; Williamson, R. T.; Christensen, M.; Schafer, W.; Welch, C. J.; ACS Cent. Sci. 2016, 2, 332.

15. Wenzel, T. J.; Chisholm, C. D.; Prog. Nucl. Magn. Reson. Spectrosc. 2011, 59, 1.

16. Nath, N.; Hebbar, S.; Prabhu, U. R.; Suryaprakash, N.; J. Indian Inst. Sci. 2010, 90, 1.

17. Seco, J. M.; Quiñoá, E.; Riguera, R.; Chem. Rev. 2012, 112, 4603.

18. Silva, M. S.; Molecules 2017, 22, 247.

19. Sharman, G. J.; eMagRes 2015, 4, 105.

20. Dale, J. A.; Mosher, H. S.; J. Am. Chem. Soc. 1968, 90, 3732.

21. Takeuchi, Y.; Itoh, N.; Kawahara, S.; Koizumi, T.; Tetrahedron 1993, 49, 1861.

22. Seco, J. M.; Latypov, S. K.; Quiñoá, E.; Riguera, R.; J. Org. Chem. 1997, 62, 7569.

23. Orlov, N. V.; Ananikov, V. P.; Green Chem. 2011, 13, 1735.

24. Pérez-Furetes, Y.; Kelly, A. M.; Fossey, J. S.; Powell, M. E.; Bull, S. D.; James, T. D.; Nat. Protoc. 2008, 3, 210.

25. Tickell, D. A.; Mahon, M. F.; Bull, S. D.; James, T. D.; Org. Lett. 2013, 15, 860.

26. Gao, J.; Srinivasan, R.; Wang, B.; Magn. Reson. Chem. 2017, $55,269$.

27. Mishra, S. K.; Suryaprakash, N. A.; RSC Adv. 2015, 5, 67277.

28. Lakshmipriya, A.; Chaudari, S. R.; Suryaprakash, N.; Chem. Commun. 2015, 51, 13492.

29. Pérez-Trujillo, M.; Castãnar, L.; Monteagudo, E.; Kuhn, L. T.; Nolis, P.; Virgili, A.; Williamson, R. T.; Parella, T.; Chem. Commun. 2014, 50, 10214.

30. Castañar, L.; Pérez-Trujillo, M.; Nolis, P.; Monteagudo, E.; Virgili, A.; Parella, T.; Chem. Phys. Chem. 2014, 15, 854.

31. Yu, S.; Pu, L.; Tetrahedron 2015, 71, 745.

32. Yi, J.; Du, G.; Yang, Y.; Li, Y.; Li, Y.; Guo, F.; Tetrahedron: Asymmetry 2016, 27, 1153.

33. Du, G.; Li, Y.; Ma, S.; Wang, R.; Li, B.; Guo, F.; Zhu, W.; Li, Y.; J. Nat. Prod. 2015, 78, 2968.

34. Ardej-Jakubisiak, M.; Kawecki, R.; Tetrahedron: Asymmetry 2008, 19, 2645.

35. Redondo, J.; Capdevila, A.; Latorre, I.; Chirality 2010, 22, 472. 
36. Yuste, F.; Sánchez-Obregón, R.; Díaz, E.; Díaz, E.; GarcíaCarrillo, M. A.; Tetrahedron: Asymmetry 2014, 25, 224.

37. Borowiecki, P.; Tetrahedron: Asymmetry 2015, 26, 16.

38. Aguilar, J. A.; Faulkner, S.; Nilsson, M.; Morris, G. A.; Angew. Chem., Int. Ed. 2010, 49, 3901.

39. Parker, D.; Taylor, R. J.; Tetrahedron 1987, 43, 5451.

40. Powell, E. M.; Kelly, A. M.; Bull, S. D.; James, T. D.; Tetrahedron Lett. 2009, 50, 876.

41. Lokesh, N.; Schin, S. L.; Narendra, L. V.; Arun, K.; Suryaprakash, N.; Org. Biomol. Chem. 2015, 13, 7230.

42. Chaudhari, S. R.; Suryaprakash, N.; J. Org. Chem. 2012, 77, 648.

43. Mishra, S. K.; Chaudhari, S. R.; Suryaprakash, N.; Org. Biomol. Chem. 2014, 12, 495.

44. Nemes, A.; Csóka, T.; Béni, S.; Farkas, V.; Rábai, J.; Szabó, D.; J. Org. Chem. 2015, 80, 6267.
45. Fukui, H.; Fukushi, Y.; Org. Lett. 2010, 12, 2856.

46. Komatsu, H.; Iwaoka, M.; Tomoda, S.; Chem. Commun. 1999, 205.

47. Nath, N.; Kumari, D.; Suryaprakash, N.; Chem. Phys. Lett. 2011, 508, 149.

48. Nath, N.; Verma, A.; Baishya, B.; Khetrapal, C. L.; Magn. Reson. Chem. 2017, 55, 553.

49. Lokesh, N.; Chaudhari, S. R.; Suryaprakash, N.; Org. Biomol. Chem. 2014, 12, 993.

50. Prabhu, U. R.; Suryaprakash, N.; J. Magn. Reson. 2010, 202, 217.

51. Castañar, L.; Parella, T.; Magn. Reson. Chem. 2015, 53, 399.

Submitted: September 17, 2017

Published online: March 1, 2018 